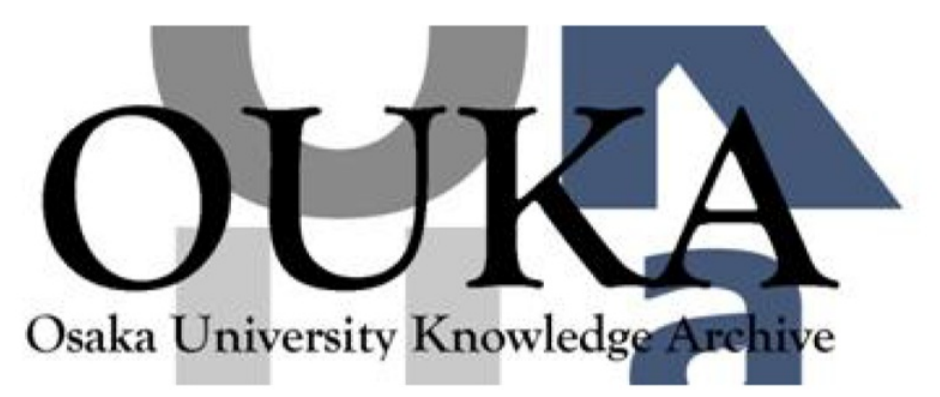

\begin{tabular}{|c|l|}
\hline Title & $\begin{array}{l}\text { Large scale analysis of interactive behaviors } \\
\text { of bubbles and particles in a liquid by a } \\
\text { coupled immersed boundary and vof technique }\end{array}$ \\
\hline Author(s) & $\begin{array}{l}\text { Iwata, Ryuichi; Takeuchi, Shintaro; Kajishima, } \\
\text { Takeo }\end{array}$ \\
\hline Citation & $\begin{array}{l}\text { Multiphase Science and Technology. 22(3) p. 233- } \\
\text { p. 246 }\end{array}$ \\
\hline Issue Date & $2010-07$ \\
\hline oaire:version & AM \\
\hline URL & https://hdl. handle. net/11094/57198 \\
\hline rights & \\
\hline Note & \\
\hline
\end{tabular}

Osaka University Knowledge Archive : OUKA

https://ir. Library. osaka-u. ac. jp/

Osaka University 


\title{
Large Scale Analysis of Interactive Behaviours of Bubbles and Particles in a Liquid by a Coupled Immersed Boundary and VOF Technique
}

\author{
Ryuichi Iwata $^{1}$, Shintaro Takeuchi ${ }^{2}$, Takeo Kajishima ${ }^{1}$ \\ 1: Dept. Mechanical Engineering, Osaka University, 2-1 Yamada-oka, Suita, Osaka, 565-0871 Japan \\ 2: Dept. Mechanical Engineering, The University of Tokyo, 7-3-1 Hongo, Bunkyo, Tokyo 113-8656 Japan
}

\begin{abstract}
A new approach for direct numerical simulation of three-phase (gas-liquid-solid) flows is proposed. Implementation of moving rigid surface in a fluid is based on an immersed boundary/solid-object method method developed by the present authors, and gas-liquid interface is captured by volume-of-fluid (VOF) method with an interface reconstruction scheme. The proposed coupling technique enables simulation of flow structures induced by both bubble and particle of comparable sizes, including the flow pattern around the gas-liquid and solid-liquid interfaces. In a suspension of 1024 solid particles and a bubble, some typical behaviours of bubble-particle interaction and liquid flow pattern are captured. The detailed analysis on the motion of the falling particles suggests that the particle rotation is strongly influenced by the behaviours of the rising bubble, giving rise a snap reversal of the rotating directions of the particles due to the flow induced by the bubble.
\end{abstract}




\section{INTRODUCTION}

Multiphase flows involving dispersed components with deformable interface are often observed in natural environment, industrial applications, biological systems, etc. The examples include bubbly flows in boiling-water reactor, petrol purification system and suspension flow of biological cells. A common problem in analysing the behaviours of the dispersed components in those systems is that it is difficult to model the dynamics of the interaction between the phases and clustered behaviours. In the present study, the authors will show recent attempts of direct numerical simulations of multiphase flows including particles and bubbles (i.e. three-phase flows) by our immersed boundary/solid-object technique coupled with volume of fluid (VOF) method.

The above systems commonly involve solid surfaces in a fluid. Implementation of moving rigid surface (moving boundary problem) in a fluid is based on a conservative momentum exchange (CMX) algorithm developed by the present authors [1;2]. In this method, the inter-phase momentum exchange is performed through the distributed interaction-force field shared by both Eulerian and Lagrangian references. This interaction algorithm offers a considerably efficient computation, and it has enabled direct numerical simulation (DNS) of interaction problem between a fluid and rigid spherical particles of a total number of $O\left(10^{3}\right)[1 ; 2]$.

For capturing the interface of multiple immiscible fluids, volume of fluid (VOF) method [3] is employed together with an interface reconstruction scheme. The authors show that the treatment for moving solid boundary in a single fluid established in the above CMX algorithm is fully applicable to a system involving interface between the immiscible fluids. In this approach, based on the one-fluid formulation, a volumeaveraged velocity field of the existing solid and fluids phases is established, and the velocity field is governed by the equation of continuity and the Navier-Stokes equation with the interaction force and surface tension. The velocity and pressure fields are time-advanced as a single continuum, irrespective of the substances (fluids or rigid object) occupying the cell. Applicability of the present method is demonstrated in a gasliquid-solid flow field involving a bubble and 1024 particles of comparable sizes to one another. Particle rotating motions in a bubble-rise flow will be studied in three-dimensions.

\section{NUMERICAL METHODS}

Throughout the present work, fluid/solid densities and viscous coefficients are assumed to be constant. 


\subsection{Basic numerical implementation for interaction between object and a fluid}

In the fluid cells partially occupied by the solid object, fluid-solid interaction is solved by a conservative momentum exchange (CMX) algorithm (for solid object immersed in an underlying fluid) proposed by Kajishima and co-researchers [1;2]. In the following, the basic idea is briefly summarised.

A velocity field $\boldsymbol{w}$ is established through volume-averaging the local fluid velocity $\boldsymbol{u}$ and the local solid phase velocity $\boldsymbol{v}$ in each cell:

$$
\boldsymbol{w}=(1-\alpha) \boldsymbol{u}+\alpha \boldsymbol{v}
$$

where $\alpha(0 \leq \alpha \leq 1)$ is the local solid volumetric fraction in the computational cell. Note that, for rigid object, $\boldsymbol{v}$ is decomposed into translating component $\boldsymbol{v}_{t}$ and rotating component $\boldsymbol{\omega} \times \boldsymbol{r}$, where $\boldsymbol{r}$ is a vector from object centre and $\omega$ is angular velocity. This one-fluid formulation in the following enables treatment of the flow field as a single continuum, and the interaction between fluid and solid at the interface can be solved through $\boldsymbol{w}$ of the following governing equations:

$$
\begin{aligned}
& \nabla \cdot \boldsymbol{w}=0, \\
& \frac{\partial \boldsymbol{w}}{\partial t}=-\frac{\nabla p}{\rho_{f}}+\boldsymbol{H}+\boldsymbol{f}_{p}, \\
& \boldsymbol{H}=-\boldsymbol{w} \cdot \nabla \boldsymbol{w}+\frac{1}{\rho_{f}} \nabla \cdot\left[\mu_{f}\left(\nabla \boldsymbol{w}+(\nabla \boldsymbol{w})^{T}\right)\right],
\end{aligned}
$$

where $p$ pressure, $\mu_{f}$ viscous coefficient, $\rho_{f}$ density of the fluid. The interaction force term $\boldsymbol{f}_{p}$ accounts for the effect of the solid object on the fluid in the cells at the interface and inside the object.

The above equations are time-updated by the same procedure as SMAC methods $[1 ; 4]$ as if a single continuum occupies the whole domain:

$$
\begin{aligned}
& \boldsymbol{w}^{*}=\boldsymbol{w}^{n}-\Delta t \frac{\nabla p^{n}}{\rho_{f}}+\frac{\Delta t}{2}\left(3 \boldsymbol{H}^{n}-\boldsymbol{H}^{n-1}\right), \\
& \nabla \cdot\left(\frac{\nabla \phi}{\rho_{f}}\right)=\frac{\nabla \cdot \boldsymbol{w}^{*}}{\Delta t} \\
& \widehat{\boldsymbol{w}}=\boldsymbol{w}^{*}-\Delta t \nabla \phi / \rho_{f}, \\
& \boldsymbol{f}_{p}=\frac{\alpha(\boldsymbol{v}-\widehat{\boldsymbol{w}})}{\Delta t} \\
& \boldsymbol{w}^{n+1}=\widehat{\boldsymbol{w}}+\Delta t \boldsymbol{f}_{p},
\end{aligned}
$$

where $\Delta t$ is a time increment and the superscripts indicate time levels. Note that in the solid cells $(\alpha=1$ and $\boldsymbol{w}=\boldsymbol{v}$ ) Eqs.(5) (8) reduce to $\boldsymbol{w}^{n+1}=\boldsymbol{v}$, while in the fluid cells ( $\alpha=0$ and $\left.\boldsymbol{w}=\boldsymbol{u}\right)$ Eqs.(5) (8) coincide with a procedure for a single-phase flow because $\boldsymbol{f}_{p}=0$. Therefore, the interaction force, Eq.(8), given at the fluid-solid interface, works to enforce the no-slip condition in the mixture field. It is also easily shown 
that $\boldsymbol{f}_{p}$ strengthens the no-permeable condition against the flux $\nabla \phi$ across the fluid-solid interface (which allows a distribution of $\phi$ within the region occupied by the solid object).

For the implementation of $\boldsymbol{f}_{p}$, solid volumetric fraction $\alpha$ needs to be evaluated in each computational cell. For simple geometries such as circular cylinder and sphere, $\alpha$ could be determined by some formulae. However, the computational load associated with this process becomes significant with the complexity of the object's geometry and as the increases in the net surface area in the whole domain. Therefore, an efficient algorithm for digitising the fluid-solid interface is of great importance. In the present study, a surface-digitiser of hyperbolic-tangent type proposed by Yuki et al. [4] is used to approximate solid volume fraction. The digitiser has been proved to be highly efficient for identifying the interface of the moving object of arbitrary geometry.

The rigid particles undergo the same interaction force with the opposite sign (i.e. repulsive force $-\boldsymbol{f}_{p}$ ), and time-advancement of the solid phase is completed by integrating the forces multiplied by the fluid's density over the volume of the particle:

$$
\begin{aligned}
& \frac{d\left(m \boldsymbol{v}_{\boldsymbol{t}}\right)}{d t}=-\int_{V_{p}} \rho_{f} \boldsymbol{f}_{\boldsymbol{p}} d V+\boldsymbol{G}_{p} \\
& \frac{d(\boldsymbol{I} \cdot \boldsymbol{\omega})}{d t}=-\int_{V_{p}} \rho_{f} \boldsymbol{r} \times \boldsymbol{f}_{\boldsymbol{p}} d V+\boldsymbol{N}_{p}
\end{aligned}
$$

where $m$ is mass of the object, $V_{p}$ region enclosing the object, $\boldsymbol{G}_{p}$ external force, $\boldsymbol{I}$ inertia moment, $\boldsymbol{N}_{p}$ external torque.

In the above formulation (Eqs.(5) (9)), the velocity field is time-updated (as the fluid) irrespective of the substance occupying the cell, which causes some discrepancy on the velocity field over the region of the rigid object. However, our further analysis shows that this discrepancy is well isolated within the region occupied by the object and that the discrepancy is overridden through updating the object velocities driven by the repulsive forces from the fluid as shown in Eqs.(10) and (11).

The use of the same interaction force for all the existing phases in a cell guarantees the momentum conservation between the phases. The volume integration of $\boldsymbol{f}_{p}$ (instead of surface integration of the surface forces) considerably reduces the computational load, which is the strong advantage of this method. This CMX algorithm has been applied to direct numerical simulation (DNS) of turbulence induced by $O\left(10^{3}\right)$ spherical particles [2] and particle clustering in a sedimentation process populating $10^{5}$ spherical particles [5]. 


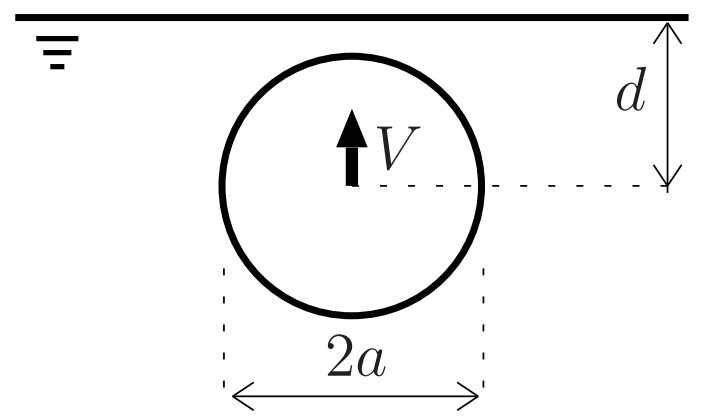

Figure 1: Schematic of a cylinder penetrating the liquid surface. The cylinder is initially placed submerged, and impulsively lifted up at velocity $V$.

\subsection{Coupling of VOF for bubble-particle interaction}

To incorporate the effect of multiple immiscible fluids on the particle motions and fluid flow structures, a volume-of-fluid (VOF) method is coupled in the present study to capture the interface between the fluids. Eq.(3) is modified to allow surface tension at the fluid-fluid interface:

$$
\frac{\partial \boldsymbol{w}}{\partial t}=-\frac{\nabla p}{\rho_{f}}+\boldsymbol{H}-\frac{\sigma \kappa \boldsymbol{n}}{\rho_{f}} \delta+\boldsymbol{f}_{g}+\boldsymbol{f}_{p}
$$

where $\sigma$ is surface tension coefficient, $\kappa$ local mean curvature of the interface, $\boldsymbol{n}$ unit vector at the interface. The gravity term $\boldsymbol{f}_{g}$ is explained in detail in Ref [6].

Volume of fluid $F$ is time-advanced by solving the following advection equation:

$$
\frac{\partial F}{\partial t}+\boldsymbol{u} \cdot \nabla F=0
$$

together with an interface-reconstruction scheme [7; 8]. Densities and viscosities in the computational cells are, respectively, given by the following local averages:

$$
\begin{aligned}
& \rho_{f}=\rho_{\ell} F+\rho_{g}(1-F) \\
& \mu_{f}=\mu_{\ell} F+\mu_{g}(1-F)
\end{aligned}
$$

where subscripts $\ell$ and $g$ denote liquid and gas, respectively. Surface tension acting at the gas-liquid interface is determined by the continuum surface force (CSF) model [9]. Eq.(6) is solved by a preconditioned Bi-CGStab method.

Although the VOF is newly coupled with the CMX algorithm, the core idea and the advantage for the momentum exchange (between a fluid and solids) remain unchanged. It should be also noted that, due to the no-permeable condition across the fluid-solid interface, no special treatment is required at a cell where the three phases exist (so long as the fluid-fluid interface is properly captured by the VOF). 


\section{VALIDATIONS}

The present method is applied to a problem involving a break-up of liquid surface due to penetration of a solid object as shown in Fig.1. Our simulation result is compared with a theoretical prediction obtained by a small-time expansion technique [10] and a numerical result reported in the literature [11]. The simulation is conducted with the same parameters as Lin [11]: the initial depth of the central axis of the cylinder is $d=1.25 a$ ( $a$ is radius of the cylinder) below the water surface and the cylinder is lifted impulsively at a constant velocity $V$. The Froude number $F r=V / \sqrt{g d}$ is 0.39 , where $g$ is gravitational acceleration. The density ratio of gas to liquid is 0.001 . A sufficiently large computational domain $(40 a \times 24 a)$, which is the same as Lin [11], is used to replicate a free space assumed in the theoretical prediction of Tyvand \& Miloh [10]. A free-slip condition is imposed on all the boundaries of the computational domain. Spatial resolution is $a / \Delta=20$ uniformly over the computational domain where $\Delta$ is grid spacing.

Sequential snapshots in Fig. 2 show that the simulated liquid surface (solid line) at normalised times $t^{*}(=t /(d / V))=0.2$ and 0.4 agree well with both the third-order theoretical prediction (broken line) by Tyvand \& Miloh [10] and the numerical solution (dotted line) by Lin [11]. At $t^{*}=0.6$, the liquid film shape of our result is still in good agreement with the numerical solution by Lin [11], whereas the theoretical prediction exhibits zero film thickness, suggesting a limitation of the asymptotic expansion in time. After breakage of the water surface $\left(t^{*} \geq 2.0\right)$, the similar surface shape to the simulation results of Lin [11] are obtained. These results indicate that our method using the simple interaction force offers as good spatial resolution as the method of Lin [11] and reasonably solves the momentum exchange between gas, liquid and solid.

We also studied the behaviour of a single bubble rising in a closed vessel of pure liquid in threedimensions. The size of the computational domain is large enough to reach terminal velocity; $13.3 D_{b}$ ( $D_{b}$ : bubble diameters) in the vertical direction and $6.7 D_{b}$ in both horizontal directions. The bubble released from the centre of the horizontal plane near the bottom. A free-slip condition is employed at all the boundaries of the computational domain. One bubble diameter is resolved by 16 meshes and the time increment is $1.0 \times 10^{-3}$. The density and viscosity ratios of gas to liquid are both set to 0.01 . Dimensionless fluid properties are Morton number $9.0 \times 10^{-7}$ and Eötvös number $3.5 \times 10^{-1}$ (corresponding Reynolds number 50 and Weber number 2). A bubble in a pure liquid is known to take a straight path under the above condition [12]. Assessing the time history of the bubble rise velocity, the difference of the simulated terminal velocity from an independently conducted simulation [13] in a body-fit coordinate system under the same Morton and Eötvös numbers is found to be less than 3\%. Our preliminary simulation employing a larger horizontal domain $\left(8 D_{b} \times 8 D_{b}\right)$ and higher resolution $\left(D_{b} / \Delta=16\right)$ shows that the terminal velocity deviate no more than $3 \%$ from the above reference data [13]. 

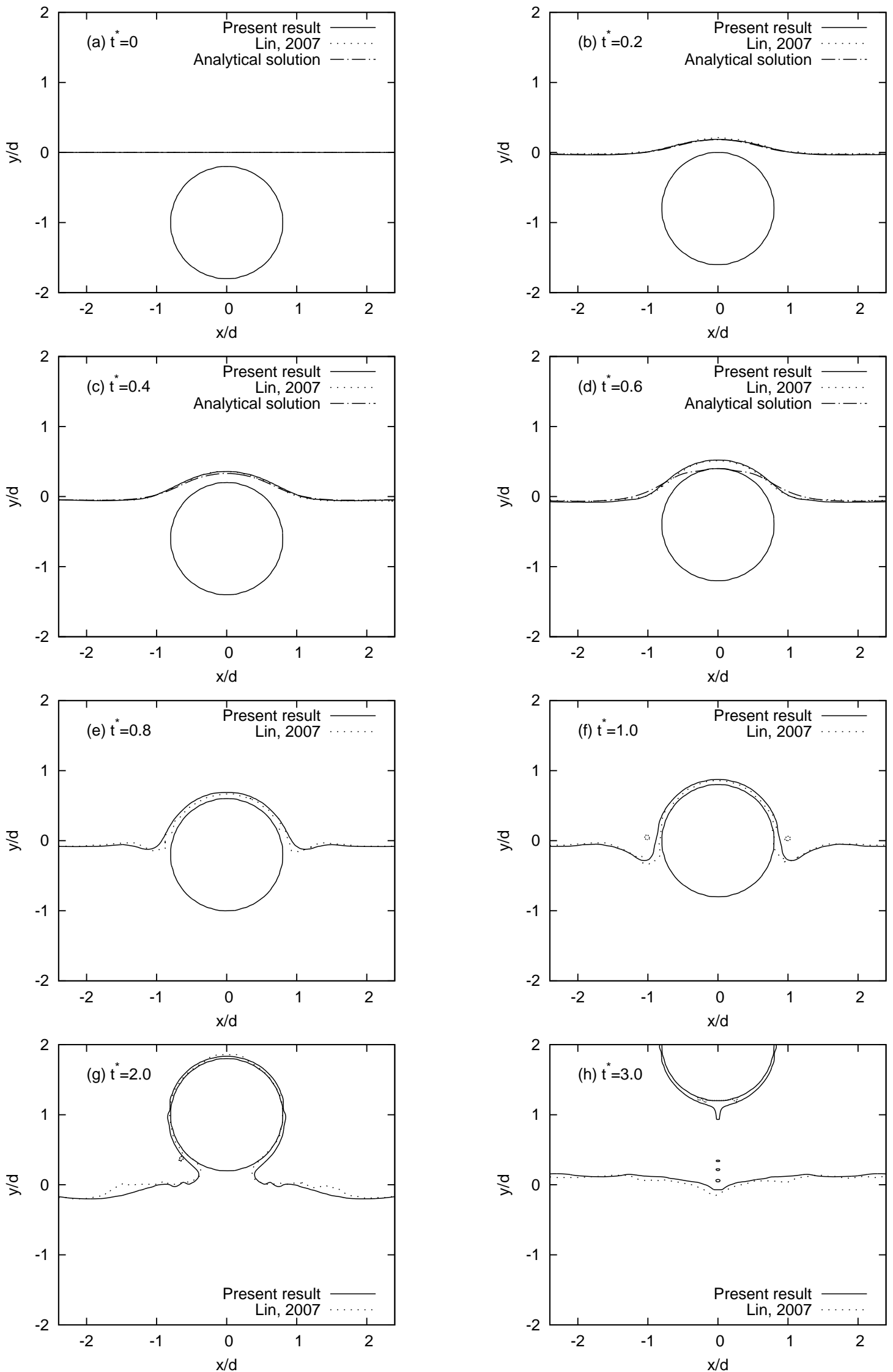

Figure 2: Sequential snapshots of a surface-breakage by a cylinder lifted up from a liquid. Solid line: present result, broken line: theoretical result by Tyvand \& Miloh [10], dotted line: numerical result by Lin [11]. 


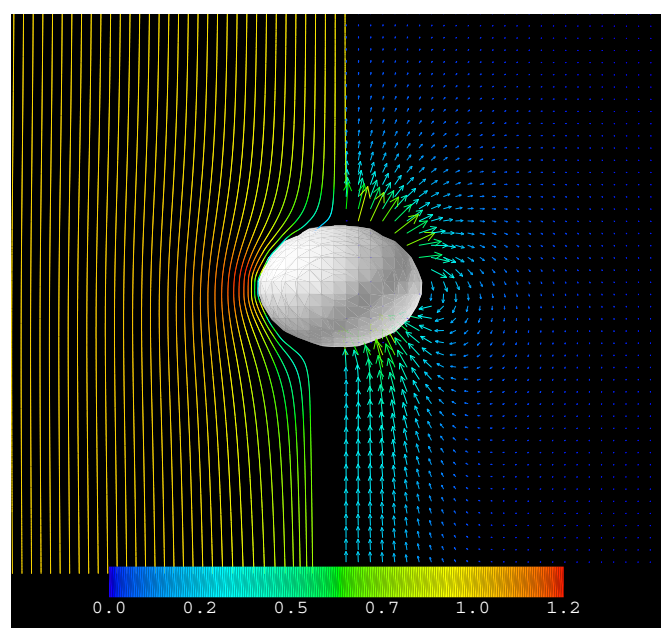

Figure 3: Cross-sectional flow field around a single bubble in steady state. Left hand side: streamlines observed from the bubble-fixed coordinate system, right hand side: velocity vectors observed from the laboratory frame.

Figure 3 shows a snapshot of a cross-sectional flow field, observed from the bubble-centred coordinate system (left) and laboratory frame (right), in a after reaching steady state. Typical flow structures involving a free-slip condition at the gas-liquid interface are observed; radially outward and inward flows (with respect to the bubble centre) in the upper and lower halves of the bubble, respectively. In the bubble wake, a typical upward current is observed.

\section{THREE-PHASE FLOW}

A large scale suspension flow of a bubble and particles is simulated.

A bubble and 1024 solid particles are initially arranged in a still liquid as shown in Fig.4. Particle size is half diameter of the initial bubble diameter. Generally, as van Sint Annaland et al. [14] suggest, with small particles (point mass), the effect of the presence of particles on the rise velocity of bubble is negligible, whereas the interaction between a bubble and particles of comparable sizes has not been studied.

The size of the computational domain is 20 bubble diameters in the vertical direction and 7.5 diameters in the horizontal directions. Density ratios of the gas and particles with respect to the liquid are, respectively, 0.01 and 2.5. The bulk volume fraction of solid in the domain is approximately 0.06. In such a dilute mixture, the inter-particle collisions are rare and unlikely to dominate the global flow field. Therefore, in the present study, a simple elastic collision model is employed. The time increment is set to $2.0 \times 10^{-3}$.

Figure 5 shows an instantaneous flow field around a contacting bubble and particle. Some characteristic behaviours of bubble-particle interaction and liquid flow pattern are captured; local counter-current flow induced by the rising bubble and descending particles. Unlike the cases studied by van Sint Annaland 


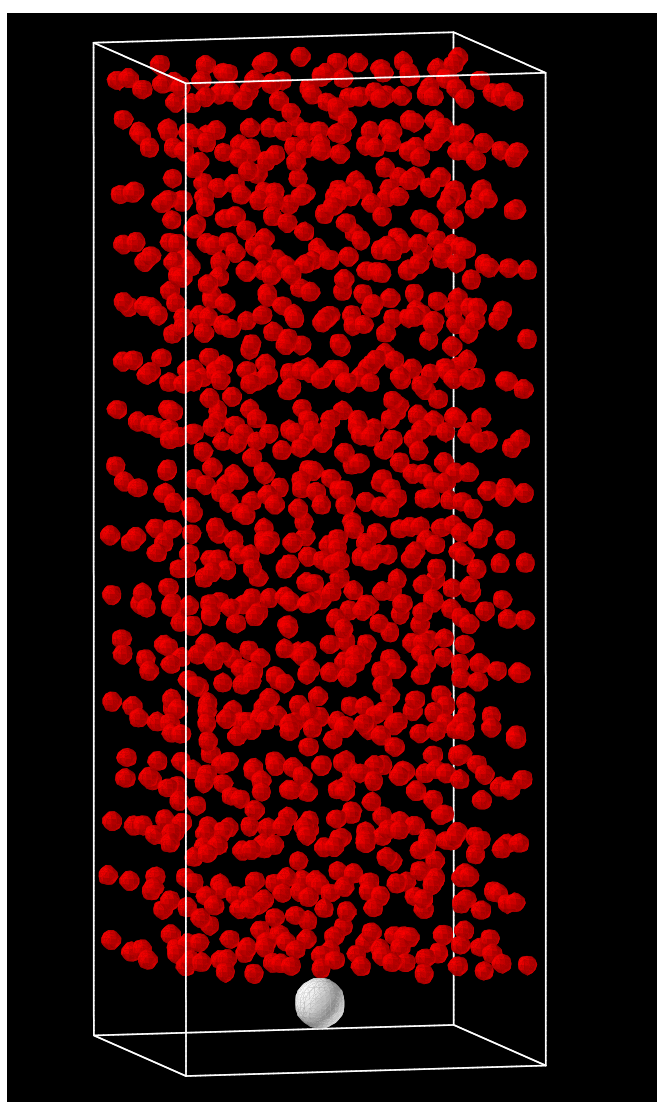

Figure 4: Initial arrangement of the particles.

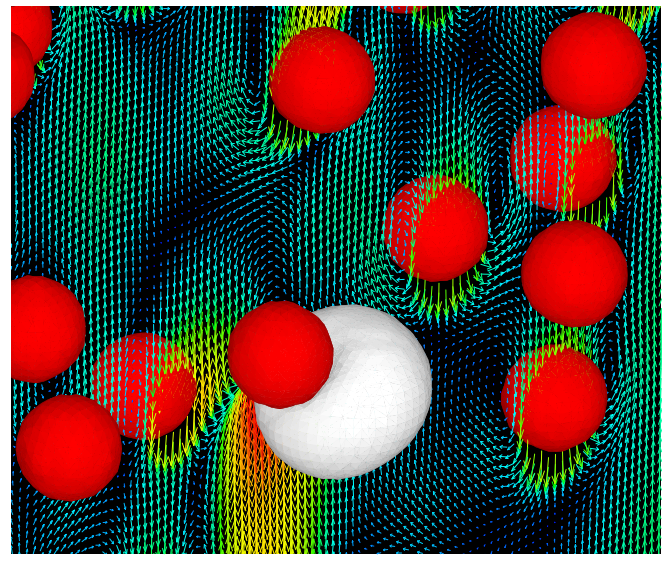

Figure 5: Instantaneous flow field around the contacting bubble and particle. Red object: particle, white iso-surface: bubble visualised by volumetric fraction of gas 0.5 . 


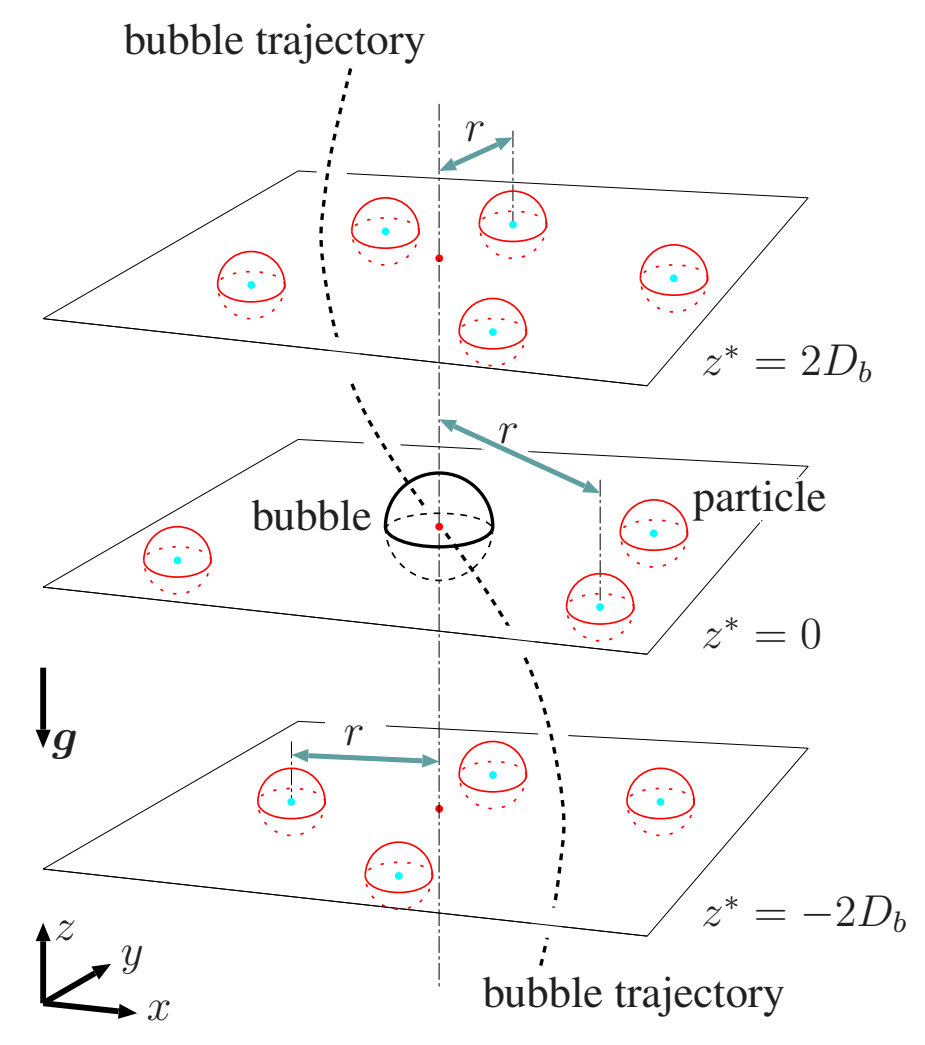

Figure 6: A 3-D schematic of horizontal planes of different vertical levels with respect to the instantaneous bubble centre.

et al. [14], because relatively large particles are employed in the present study, the bubble rise motion is decelerated due to the blockage by the particles, causing horizontal motion of the bubble through the interparticle gaps.

To see the effect of bubble on the particle motion, particle behaviours are averaged over different horizontal planes. Vertical (signed) distance measured from the bubble centre is denoted by $z^{*}=\left(z-z_{b}\right) / D_{b}$, where $z_{b}$ is the vertical coordinate of the centroid of the bubble and $D_{b}$ is the initial bubble diameter. Some horizontal planes are arranged as shown in Fig.6. Figure 7 illustrates the local coordinates $(r, \theta)$ for each particle in a horizontal plane.

In Fig.8, the radial distributions of particle rotations before and after the passage of the bubble are compared by averaged azimuthal component of the particle angular velocity, $\overline{\omega_{\theta}^{*}}=\omega_{\theta} / \sqrt{g / D_{b}}$. Note that the radius is normalised by the particle diameter and denoted by $r^{*}$.

For the particles at the levels higher than $z^{*}=0.5$, the upward component of radially outward flow, as observed in Fig.3, supplies positive angular momentum around $\theta$ axis. In the region right above the bubble $\left(z^{*}=0.5\right)$, the radially outward flow may contribute to horizontal migration and counter rotation of the particles. From our further observations, the obvious tendency of radially-outward movement of the 


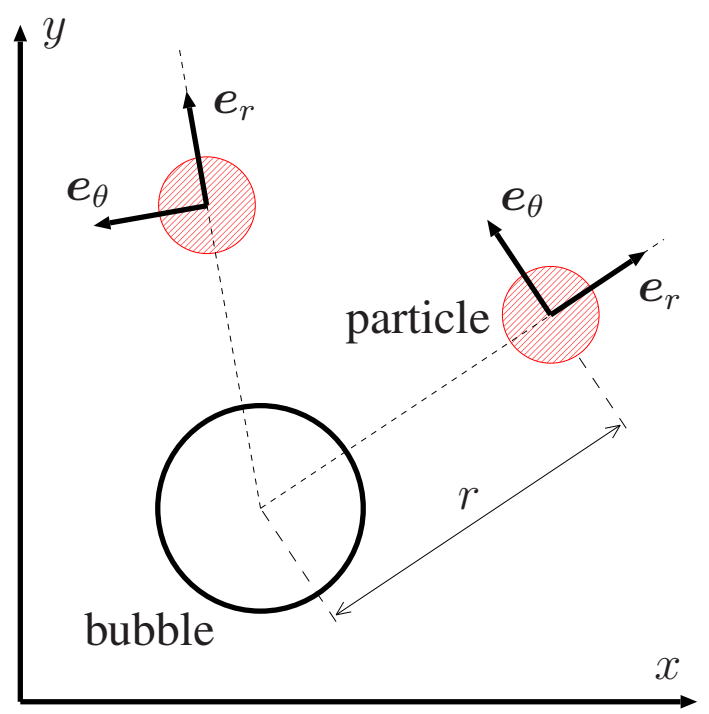

Figure 7: A horizontal view of the local coordinate system $(r, \theta)$ with respect to each particle. A radial unit vector $\boldsymbol{e}_{r}$ is define along the line from the bubble centre axis to particle centre within the plane, and $\boldsymbol{e}_{\theta}$ is a unit tangential vector in the same plane.

particles is recognised at this level. However, most particles still keep rotating in the positive direction probably due to the inertia.

On the other hand, at $z^{*}=0.3$, the angular velocity exhibits a reversal of sign in the vicinity of the bubble due to the negative angular momentum transferred from the strong outward flow acting on the lower hemispheres of the particles. As the particle passes by the bubble centre $\left(z^{*}=0\right)$, the particle rotation is further accelerated. By the time particle travels down to $z^{*}=-0.3$ and -0.5 , however, the radially inward flow contributes to a slight increase in average particle angular velocity (but not as much increase as the decrease observed between $z^{*}=0$ and 0.3). After these levels $\left(z^{*}=-0.5 \sim-3.0\right)$, the rotation of the particles attenuates gradually due to the viscous effect and the upward current induced by the bubble.

The above results suggest that a bubble and particles, of comparable sizes to one another, show strong interaction through a background fluid and that the particle rotation is particularly influenced by the behaviours and the near-surface flow of the bubble.

\section{CONCLUDING REMARKS}

Direct numerical simulation methods have been developed to analyse multiphase flows involving dispersed components with deformable interfaces (i.e., gas-liquid-solid three-phase flow) by incorporating the volume-of-fluid method into our conservative momentum exchange (CMX) algorithm for solid objects immersed in underlying fluids. 


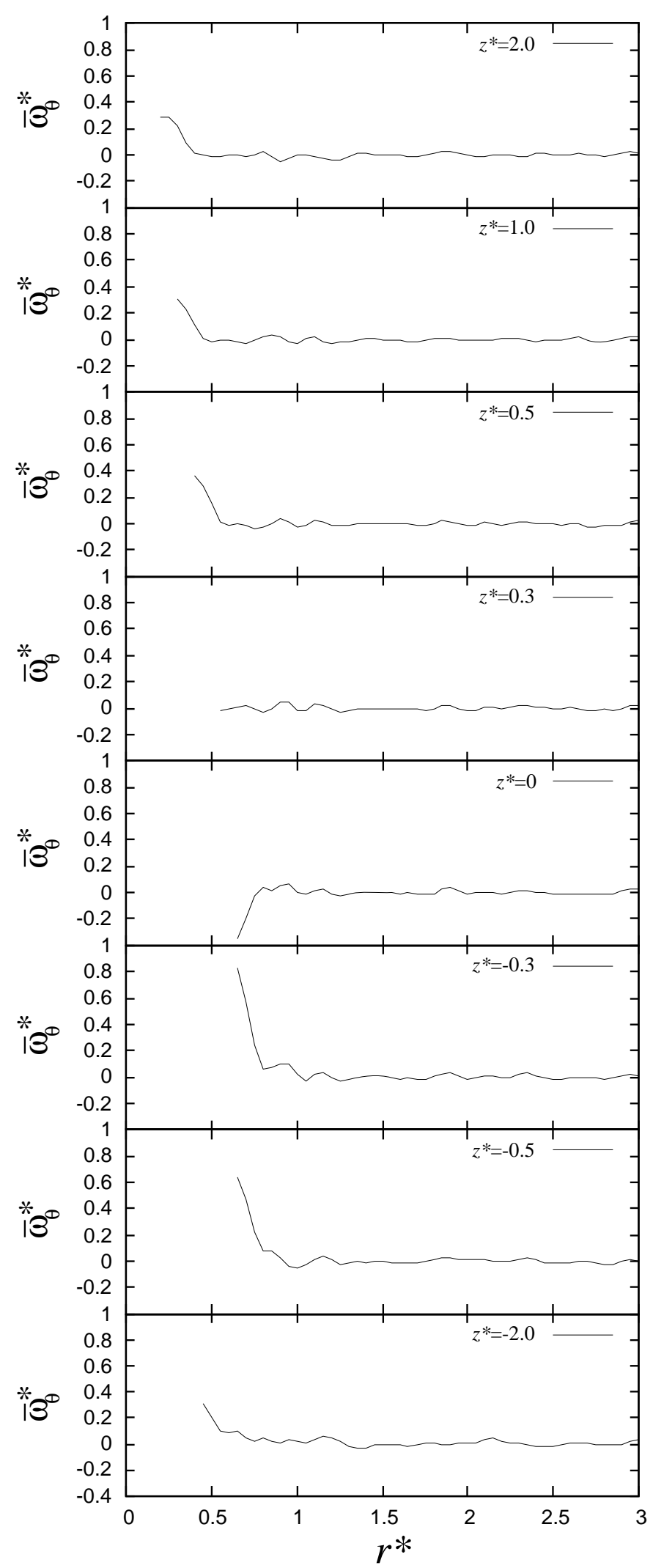

Figure 8: Time evolution of radial distributions of the averaged $\theta$-component of the particle angular velocity. The radius and angular velocity are, respectively, normalised by the particle diameter $D_{b}$ and $\sqrt{g / D_{b}}$. 
The flow structures around the individual dispersed phases (bubble and particle) are resolved, and the unique fluid-solid coupling technique has enabled incorporation of a large population of finite-sized solid objects (comparable to the bubble size).

The methods promise to be unique engineering tools available for many challenging problems, especially for fluid-solid interaction problems involving immiscible fluids. 


\section{NOMENCLATURE}

$\begin{array}{ll}D_{b} & \text { bubble diameter } \\ F & \text { volume of fluid } \\ F r & \text { Froude number } \\ \boldsymbol{G}_{p} & \text { external force } \\ I & \text { inertia moment } \\ \boldsymbol{N}_{p} & \text { external torque } \\ V & \text { traction velocity } \\ V_{p} & \text { region enclosing solid object } \\ a & \text { cylinder radius } \\ d & \text { depth } \\ \boldsymbol{e}_{\theta} & \text { unit vector in tangential direction } \\ \boldsymbol{e}_{r} & \text { unit vector in radial direction } \\ \boldsymbol{f}_{p} & \text { interaction force } \\ g & \text { gravitational acceleration } \\ m & \text { mass } \\ \boldsymbol{n} & \text { unit vector } \\ p & \text { pressure } \\ r & \text { radial coordinate } \\ \boldsymbol{r} & \text { position vector } \\ r^{*} & \text { normalised radius } \\ \boldsymbol{u} & \text { fluid velocity } \\ \boldsymbol{v} & \text { particle velocity } \\ \boldsymbol{v}_{t} & \text { translating component of } \boldsymbol{v} \\ \boldsymbol{w} & \text { volume-averaged velocity } \\ x, y, z & \text { Cartesian coordinates } \\ z^{*} & \text { normalised vertical level } \\ z_{b} & \text { vertical level of bubble centroid }\end{array}$

Greek

$\alpha \quad$ local solid volumetric fraction

$\Delta \quad$ grid spacing

$\Delta t \quad$ time increment

$\theta \quad$ azimuthal coordinate

$\kappa \quad$ local mean curvature

$\mu_{f} \quad$ viscous coefficient

$\sigma \quad$ is surface tension coefficient

$\rho_{f} \quad$ fluid density

$\phi \quad$ pressure potential

$\omega \quad$ angular velocity

$\omega_{\theta} \quad$ azimuthal component of angular velocity

\section{$\underline{\text { Superscript }}$}

$n$

time level

$\underline{\text { Subscript }}$

\begin{tabular}{ll}
\hline & liquid \\
$g$ & gas
\end{tabular}




\section{References}

1 Kajishima, T., Takiguchi, S., Hamasaki, H. \& Miyake, Y., "Turbulence structure of particle-laden flow in a vertical plane channel due to vortex shedding", JSME Int. J. Ser. B 44-4, 526-535 (2001)

2 Kajishima, T. \& Takiguchi, S., "Interaction between particle clusters and fluid turbulence”, Int. J. Heat and Fluid Flow 23(5), 639-646 (2002)

3 Hirt, C. W. \& Nichols, B. D., "Volume of Fluid (VOF) Method for the Dynamics of Free Boundaries", J. Comput. Phys. 39, 201-225, 1981

4 Yuki, Y., Takeuchi, S. \& Kajishima, T. ”Efficient Immersed Boundary Method for Strong Interaction Problem of Arbitrary Shape Object with the Self-Induced Flow" Journal of Fluid Science and Technology 2-1, 1-11 (2007)

5 Nishiura, D., Shimosaka, A., Shirakawa, Y. \& Hidaka, J. ”Particle Behaviours under Sedimentation by Hybrid Simulation of Discrete Element Method and Direct Numerical Simulation" (in Japanese) Trans. Soc. Chem. Eng. Japan 32-4, 331-340 (2006)

6 Iwata, R., Kajishima, T. and Takeuchi, S. "Analysis of a rising bubble passing through array of particles by a coupled immersed-boundary and volume-of-fluid method" (in Japanese), Japanese Journal of Multiphase Flow Vol.23 No.5, pp.531-538 (2010)

7 Aulisa, E., Manservisi, S., Scardovelli \& R., Zaleski, S., 2007. Interface reconstruction with leastsquares fit and split advection in three-dimensional Cartesian geometry. J. Comput. Phys. 225-2, 23012319.

8 Gueyffier, D., Li, J., Nadim, A., Scardovelli, R. \& Zaleski, S., 1999. Volume-of-fluid interface tracking with smoothed surface stress methods for three dimensional flows. J. Comput. Phys. 152, 423-456.

9 Brackbill, J.U., Kothe, D.B. \& Zemach, C., 1992. Method for modeling surface tension, J. Comput. Phys. 100, 335-354.

10 Tyvand, P. \& Miloh, T., "Free-surface flow due to impulsive motion of a submerged circular cylinder" J. Fluid Mech. 286, 67-101 (1995)

11 Lin, P., "A fixed grid simulation of a moving body in free surface flows", Comput. Fluids 36, 549-561 (2007) 
12 Magnaudet, J. \& Eames, I., "The motion of high-Reynolds-number bubbles in inhomogeneous flow". Annu. Rev. Fluid Mech. 32, 659-708 (2000)

13 Ryskin, G. \& Leal, L.G., 1984. Numerical solution of free-boundary problems in fluid mechanics. Part 2. Buoyancy-driven motion of a gas bubble through a quiescent liquid. J. Fluid Mech. 148, 19-35.

14 van Sint Annaland, M., Deen, N.G. \& Kuipers. J.A.M., 2005. Numerical simulation of gas-liquid-solid flows using a combined front tracking and discrete particle method. Chemical Engineering Science 60, 6188-6198.

\section{Acknowledgement}

One of the authors, R.I., gratefully acknowledges the research grant of the Japan Society for the Promotion of Science (JSPS), Grant No.21.496 (2009-2010). 